\title{
ULTIMATE STATE BOUNDEDNESS OF UNDERACTUATED SPACECRAFT SUBJECT TO AN UNMATCHED DISTURBANCE
}

\author{
Rouzbeh Moradi, Alireza Alikhani \\ Aerospace Research Institute (Ministry of Science, Research and Technology), Tehran, Iran \\ e-mail: roozbeh_moradi_aerospace@yahoo.com; aalikhani@ari.ac.ir (correspondingauthor) \\ Mohsen FAThi Jegarkandi \\ Department of Aerospace Engineering, Sharif University of Technology, Tehran, Iran \\ e-mail: fathi@sharif.edu
}

\begin{abstract}
Ultimate state boundedness for underactuated spacecraft subject to large non-matched disturbances is attained. First, non-smooth time-invariant state feedback control laws that make the origin asymptotically stable are obtained. Then, the controller is extended to make the closed-loop system globally uniformly ultimately bounded under the following conditions: 1) the disturbances acting on the directly actuated states are known and 2) the disturbance acting on the unactuated state is bounded and its profile need not be known. Finally, numerical simulations are presented to verify the analytical results. A large step disturbance is considered, and it is shown that the proposed controller makes the closed-loop system globally uniformly ultimately bounded. The proposed method is rather general and can be extended to other systems.
\end{abstract}

Keywords: underactuated spacecraft stabilization, non-matched disturbances, global uniform ultimate boundedness

\section{Introduction}

A mechanical system is underactuated when the number of independent control inputs is less than the number of degrees of freedom to be controlled. Considering the stabilization of such systems, an extensive amount of studies has been published in the literature. Choukchou-Braham et al. (2013), Olfati-Saber (2001), Aneke (2003), Spong (1998), Fantoni and Lozano (2002) and Liu and Yu (2013) are just a few examples.

The linearized model of a majority of underactuated systems, especially in the absence of gravitational terms, is not controllable near equilibrium points (Choukchou-Braham et al., 2013). This leads to the well-known fact that most underactuated systems do not satisfy Brockett's necessary condition for smooth feedback stabilization (Choukchou-Braham et al., 2013). In order to deal with this problem, non-smooth feedbacks have been proposed to stabilize underactuated mechanical systems (Reyhanoglu et al., 2000).

In addition to the smoothness of feedback control laws, underactuation leads to another important challenge: attenuation of disturbances. The severity of this problem increases when the disturbances are non-matched, i.e. $\operatorname{span}\{P\} \notin \operatorname{span}\{B\}$, where $P$ and $B$ are the disturbance and control matrices, respectively (Astolfi and Rapaport, 1998).

Reducing the effects of disturbances on the stabilization of underactuated spacecraft has been considered by several papers. Astolfi and Rapaport (1998) considered robust stabilization of the angular velocity of a rigid body subject to external disturbances using $L_{2}$-gain analysis. Several propositions were proved, and the robust stabilization problem was solved in a region having a hole. Floquet et al. (2000) used higher order sliding mode control (variable structure 
control) to make the origin asymptotically stable for underactuated spacecraft within a finite time. However, it was assumed that no disturbance was exerted on the unactuated axis, i.e. they satisfied matching conditions. Karami and Sassani (2000) used the backstepping technique to asymptotically stabilize the angular velocity and attitude of underactuated spacecraft. The considered step disturbance had a very low magnitude. Zhang et al. (2008) considered the spin stabilization problem of underactuated spacecraft subject to sinusoidal disturbances. All of the disturbances were considered to be sinusoidal. Wang et al. (2003) considered stabilization of the angular velocity and attitude of underactuated spacecraft under sinusoidal disturbance. Although three exogenous disturbances were considered to be exerted on the spacecraft, the controller design was based on matched type disturbances.

In this paper, a new method is proposed to find non-smooth time-invariant state feedback control laws for underactuated spacecraft. Then, the controller is extended to make the closedloop system globally uniformly ultimately bounded (GUUB). The proposed method is based on a combination of feedback linearization and Lyapunov stability theory. To avoid singularity of control inputs near the equilibrium points, a thin boundary layer is defined. It is assumed that outside the boundary layer, the extended controller is applied to the system. However, inside the boundary layer, the terms leading to singularity are canceled.

The present paper has two contributions: First, the proposed method is rather general and can be extended to other systems and second, the large step disturbance is considered to verify the controller performance. Most of the previous works have considered sinusoidal disturbances, which are not as severe as the step disturbance for the underactuated spacecraft.

The rest of this paper consists of the following sections: Section 2 provides non-smooth time-invariant state feedback control of underactuated spacecraft. In Section 3, the controller is extended to make the closed-loop system GUUB subject to non-matched disturbances. Finally, Section 4 presents numerical simulations and discussions to verify the analytical results.

\section{Time-invariant non-smooth state feedback control of underactuated spacecraft}

In the principal coordinate system, the rigid spacecraft angular velocity equations are described by the following expressions (Sidi, 2000)

$$
\begin{array}{ll}
\dot{p}=\alpha_{1} q r+u_{1}+d_{p} & d_{p}=\frac{T_{d p}}{J_{x}} \\
\dot{q}=\alpha_{2} p r+u_{2}+d_{q} & d_{q}=\frac{T_{d q}}{J_{y}} \\
\dot{r}=\alpha_{3} p q+u_{3}+d_{r} & d_{r}=\frac{T_{d r}}{J_{z}}
\end{array}
$$

where $[p, q, r]$ are angular velocities of the spacecraft, $\left[u_{1}, u_{2}, u_{3}\right]$ are normalized control inputs and $\left[T_{d p}, T_{d q}, T_{d r}\right]$ are external disturbances. $\alpha_{1}, \alpha_{2}$ and $\alpha_{3}$ are fractions of moments of inertia and are assumed to be constant. Their values are obtained from the following set of equations

$$
\alpha_{1}=\frac{J_{y}-J_{z}}{J_{x}} \quad \alpha_{2}=\frac{J_{z}-J_{x}}{J_{y}} \quad \alpha_{3}=\frac{J_{x}-J_{y}}{J_{z}}
$$

where $\left[J_{x}, J_{y}, J_{z}\right]$ are principal moments of inertia of the rigid body along the principal body axis. The relation between control moments and inputs are given by the following equations

$$
u_{1}=\frac{M_{x}}{J_{x}} \quad u_{2}=\frac{M_{y}}{J_{y}} \quad u_{3}=\frac{M_{z}}{J_{z}}
$$

$\left[M_{x}, M_{y}, M_{z}\right]$ are three control moments acting on the spacecraft, and are assumed to be produced by thrusters. 
Without loss of generality, it is assumed that the third state $(r)$ is unactuated $\left(u_{3}=0\right)$. On the other hand, it is assumed that $\alpha_{i} \neq 0 \forall i=1,2,3$.

For now, the disturbances are not considered in the controller design. Therefore, Eqs. (2.1) will be simplified to Eqs. (2.4)

$$
\dot{p}=\alpha_{1} q r+u_{1} \quad \dot{q}=\alpha_{2} p r+u_{2} \quad \dot{r}=\alpha_{3} p q
$$

In order to obtain a virtual control input for $r, q$ is bisected into two parts

$$
q=a q_{1}+b q_{2} \quad a, b \in R
$$

$a$ and $b$ are constant real numbers that are presented to show that any linear combination of $q_{1}$ and $q_{2}$ will lead to the same results. This fact will be confirmed shortly.

Inserting $q$ into the second and third rows of Eqs. (2.4) leads to

$$
\dot{p}=\alpha_{1} q r+u_{1} \quad a \dot{q}_{1}=\alpha_{2} p r+u_{2}-b \dot{q}_{2} \quad \dot{r}=\alpha_{3} p\left(a q_{1}+b q_{2}\right)
$$

The following definition is introduced

$$
\dot{q}_{2}=w
$$

$w$ is a scalar variable that is used to stabilize the unactuated state $(r)$. The goal is now to determine $q_{2}$ that forces $r$ to approach the origin. This $q_{2}$ is denoted as $q_{2, \text { des }}$.

In order for $r$ to be exponentially stabilized, the following relation must hold

$$
\dot{r}=-k_{r} r
$$

Considering the last row of Eqs. (2.6) and (2.8), the following equation is obtained

$$
-k_{r} r=\alpha_{3} p\left(a q_{1}+b q_{2, d e s}\right)
$$

Solving for $q_{2, \text { des }}$ results in

$$
q_{2, \text { des }}=\frac{1}{b} \phi-\frac{a}{b} q_{1}
$$

where $\phi=-k_{r} r /\left(\alpha_{3} p\right)$.

It can be easily shown that

$$
\lim _{t \rightarrow \infty} q=\lim _{t \rightarrow \infty} \phi
$$

Therefore, it is possible to tune the controller parameters to make sure that the steady-state value of $q$ becomes zero.

Now, the following linear combination of $q_{2}$ and $q_{2, \text { des }}$ is introduced to transform Eqs. (2.6) into a virtually fully actuated form

$$
z=c q_{2}+d q_{2, \text { des }} \quad c, d \in R
$$

$c$ and $d$ are constant real numbers, and their values have direct influence on the stability and performance of the closed-loop system. The validity and importance of this statement will become clear at the end of this Section. As stated previously, $q_{2, \text { des }}$ is the virtual control input.

Note 1: If $c=-d$ and $z=0, q_{2}$ will be equal to $q_{2, d e s}$, which is the ideal case. This is equivalent to saying that the stabilization of $z$ is equivalent to the stabilization of $r$. According to this point, the equation for $\dot{r}$ will be replaced by $\dot{z}$. 
Differentiating Eq. (2.12) with respect to time together with Eq. (2.7) results in

$$
\dot{z}=c \dot{q}_{2}+d \dot{q}_{2, d e s}=c w+d \dot{q}_{2, d e s}
$$

Replacing the third equation of Eqs. (2.6) with Eq. (2.13) leads to

$$
\dot{p}=\alpha_{1} q r+u_{1} \quad \dot{q}_{1}=\frac{1}{a}\left(\alpha_{2} p r+u_{2}-b \dot{q}_{2}\right) \quad \dot{z}=c w+d \dot{q}_{2, d e s}
$$

The time derivative of $q_{2, \text { des }}$ can be obtained by partial differentiation of Eq. (2.10)

$$
\dot{q}_{2, \text { des }}=\frac{1}{b} \frac{\partial \phi}{\partial p} \dot{p}-\frac{a}{b} \dot{q}_{1}
$$

It is assumed that $(\partial \phi / \partial r) / \dot{r} \approx 0$ in comparison to the other terms. The reason for this assumption is to rewrite $\dot{z}$ in terms of the original variables.

Using Eqs. (2.5), (2.7), (2.15) and performing some mathematical operations, along with the third row of Eqs. (2.4), $\dot{z}$ will be simplified to

$$
\dot{z}=(c+d) w+\frac{d}{b} \frac{\partial \phi}{\partial p} \dot{p}-\frac{d}{b} \dot{q}
$$

Therefore, the entire set of Eqs. (2.14) in a virtually fully actuated form will be given as

$$
\dot{p}=\alpha_{1} q r+u_{1} \quad \dot{q}_{1}=\frac{1}{a}\left(\alpha_{2} p r+u_{2}-b w\right) \quad \dot{z}=(c+d) w+\frac{d}{b} \frac{\partial \phi}{\partial p} \dot{p}-\frac{d}{b} \dot{q}
$$

According to the third row, in order for $z$ to be stabilizable, $c$ should not be equal to $-d$. However, this is in contradiction with the previously made conclusion (Note 1). In order to alleviate this problem, it will be assumed that $c \approx-d$.

Using feedback linearization and expecting exponential convergence from $z$ i.e. $\dot{z}=-k_{z} z$, $w$ is obtained as follows

$$
w=\frac{1}{c+d}\left(-k_{z} z-\frac{d}{b} \frac{\partial \phi}{\partial p} \dot{p}+\frac{d}{b} \dot{q}\right)
$$

Inserting $w$ in the second equation of Eqs. (2.17), $u_{2}$ is obtained as follows

$$
u_{2}=a \dot{q}_{1}-\alpha_{2} p r+\frac{b}{c+d}\left(-k_{z} z-\frac{d}{b} \frac{k_{p} k_{r}(-r)}{\alpha_{3} p}-\frac{d}{b} k_{q} q\right)
$$

Using Eq. (2.12) and considering the fact that $\lim _{t \rightarrow \infty} q_{2, \text { des }}=0$, the above equation is simplified to

$$
u_{2}=a \dot{q}_{1}-\alpha_{2} p r+\frac{b}{c+d}\left(-k_{z} c q_{2}-\frac{d}{b} \frac{k_{p} k_{r}(-r)}{\alpha_{3} p}-\frac{d}{b} k_{q} q\right)
$$

The reason for assuming $\lim _{t \rightarrow \infty} q_{2, \text { des }}=0$ can be inferred from Eq. (2.10). $\phi$ is a function that can converge to zero through tuning the controller parameters $\left(k_{p}<k_{r}\right.$ or equivalently, the convergence rate of $p$ less than the convergence rate of $r$ ). On the other hand, according to the dynamics imposed on $q_{1}\left(\dot{q}_{1}=-k_{q_{1}} q_{1}\right)$, this variable will also converge to zero. Considering these facts, $u_{2}$ is

$$
u_{2}=-a k_{q_{1}} q_{1}-\alpha_{2} p r-\frac{b}{c+d} k_{z} c q_{2}-\frac{d}{c+d} \frac{k_{p} k_{r}(-r)}{\alpha_{3} p}-\frac{d}{c+d} k_{q} q
$$


Assuming $k_{q_{1}}=[c /(c+d)] k_{z}=[c /(c+d)] k_{q}$ and according to Eq. (2.5), the final equation for $u_{1}$ and $u_{2}$ is

$$
u_{1}=-k_{p} p-\alpha_{1} q r \quad u_{2}=-k_{q} q-\alpha_{2} p r+\frac{d}{c+d} \frac{k_{p} k_{r} r}{\alpha_{3} p}
$$

Since the procedure used to obtain this controller is based on linear state bisection (Eq. (2.5)), this controller is called LSB.

In accordance with Eq. (2.22), $u_{2}$ consists of two parts

$$
u_{2_{1}}=-k_{q} q-\alpha_{2} p r \quad u_{2_{2}}=\frac{d}{c+d} \frac{k_{p} k_{r} r}{\alpha_{3} p}
$$

$u_{2_{1}}$ and $u_{2_{2}}$ are used to stabilize $q$ and $r$, respectively. The form of $u_{2}$ is consistent with the one proposed by Reyhanoglu (1996).

According to Eqs. (2.23), $u_{2}$ does not depend on $a$ and $b$. According to Eq. (2.1), the components of $d_{p}$ and $d_{q}$ can be easily counteracted by the direct control input vectors, $u_{1}$ and $u_{2}$. Therefore, the component of disturbances on the unactuated state $\left(d_{r}\right)$ plays the key role in the controller design.

Non-smooth time-invariant state feedback control laws (Eq. (2.22)) make the origin asymptotically stable for the disturbance-free system (Eqs. (2.4)). In the next Section, this controller is extended to provide GUUB, in the presence of non-matched disturbances.

\section{Underactuated spacecraft angular velocity ultimate boundedness in presence of non-matched disturbances}

Definition 1 (Astolfi and Rapaport (1998)): The disturbances are non-matched when $\operatorname{span}\{P\} \notin \operatorname{span}\{B\}$, where $P$ and $B$ are the disturbance and control matrices, respectively.

Assumption 1: $d_{p}$ and $d_{q}$ can be unbounded, but should be known.

Assumption 2: $d_{r}$ should be bounded, and its maximum value should be known.

In order to extend the controller and to ensure that the states are GUUB, the following procedure is proposed:

1) Since $u_{2}$ has been selected to stabilize $r, u_{1}$ is used to provide GUUB.

2) A candidate Lyapunov function (CLF) is proposed.

3) The derivative of this CLF along the trajectories of the closed-loop system is evaluated.

4) $u_{1}$ is used to ensure GUUB of the states for the perturbed closed-loop system.

Consider Eqs. (2.4) with disturbances

$$
\dot{p}=u_{1}+\alpha_{1} q r+d_{p} \quad \dot{q}=u_{2}+\alpha_{2} p r+d_{q} \quad \dot{r}=\alpha_{3} p q+d_{r}
$$

It has been shown in Section 2 that $u_{1}$ and $u_{2}$ (Eq. (2.22)) make the origin asymptotically stable for Eqs. (3.1) without the presence of disturbances.

After eliminating $d_{p}$ and $d_{q}$ using direct control inputs, the closed-loop system will be

$$
\dot{p}=u_{1}^{\prime} \quad \dot{q}=-k_{q} q+\frac{d}{c+d} \frac{k_{p} k_{r} r}{\alpha_{3} p} \quad \dot{r}=\alpha_{3} p q+d_{r}
$$

Now, the goal is to find $u_{1}^{\prime}$ that makes the closed-loop system GUUB. 
In order to solve this problem, the following CLF is proposed

$$
V=\frac{1}{2}\left[\begin{array}{lll}
p & q & r
\end{array}\right]\left[\begin{array}{ccc}
k_{p} & 0 & 0 \\
0 & k_{q} & 0 \\
0 & 0 & k_{r}
\end{array}\right]\left[\begin{array}{l}
p \\
q \\
r
\end{array}\right]=\frac{1}{2}\left(k_{p} p^{2}+k_{q} q^{2}+k_{r} r^{2}\right)
$$

The derivative of $V$ along the trajectories (Eqs. (3.2)) is given by

$$
\begin{aligned}
\dot{V}= & \frac{\partial V}{\partial x} \dot{x}=k_{p} p u_{1}^{\prime}-k_{q}^{2} q^{2}+\frac{d}{c+d} \frac{k_{p} k_{q} k_{r}}{\alpha_{3}} \frac{r q}{p}+\alpha_{3} k_{r} p q r+k_{r} r d_{r} \\
& \leqslant k_{p} p u_{1}^{\prime}-k_{q}^{2} q^{2}+\frac{d}{c+d} \frac{k_{p} k_{q} k_{r}}{\alpha_{3}} \frac{r q}{p}+\alpha_{3} k_{r} p q r+k_{r} r M_{d}
\end{aligned}
$$

where $M_{d}$ is the maximum absolute value of $d_{r}$. If $u_{1}^{\prime}$ is selected as

$$
u_{1}^{\prime}=\frac{1}{k_{p} p}\left(-\frac{d}{c+d} \frac{k_{p} k_{q} k_{r}}{\alpha_{3}} \frac{r q}{p}-\alpha_{3} k_{r} p q r-k_{p}^{2} p^{2}-k_{r}^{2} r^{2}\right) \quad p \neq 0
$$

$\dot{V}$ will result in the following equation

$$
\dot{V} \leqslant-k_{p}^{2} p^{2}-k_{q}^{2} q^{2}-k_{r}^{2} r^{2}+k_{r} r M_{d}=-w_{2}(x)+k_{r} r M_{d}
$$

Since

$$
-w_{2}(x) \leqslant-\min \left(k_{p}^{2}, k_{q}^{2}, k_{r}^{2}\right)\|x\|_{2}^{2}
$$

and at the same time $r \leqslant\|x\|_{2}, \dot{V}$ will satisfy the following inequality

$$
\dot{V} \leqslant-\min \left(k_{p}^{2}, k_{q}^{2}, k_{r}^{2}\right)\|x\|_{2}^{2}+k_{r} M_{d}\|x\|_{2}
$$

Therefore, if the following inequality holds

$$
M_{d} \leqslant \frac{\min \left(k_{p}^{2}, k_{q}^{2}, k_{r}^{2}\right)}{2 k_{r}}\|x\|_{2}
$$

equivalently

$$
\|x\|_{2} \geqslant \frac{2 k_{r}}{\min \left(k_{p}^{2}, k_{q}^{2}, k_{r}^{2}\right)} M_{d}
$$

$\dot{V}$ will satisfy the following relation

$$
\dot{V} \leqslant-\frac{1}{2} \min \left(k_{p}^{2}, k_{q}^{2}, k_{r}^{2}\right)\|x\|_{2}^{2}=-w_{1}(x)
$$

where $w_{1}(x)$ is a positive definite function. Equations (3.3) and (3.11) confirm that the conditions of theorem 4.18 (Khalil, 2001) are satisfied. This means that the states of Eqs. (3.2) become GUUB for the state-feedback control law given by Eq. (3.5).

An important parameter is introduced: $t h p_{0}$ or the thicknesses of the boundary layer. This parameter is selected such that the control inputs never reach the singular point and. at the same time, the states reach the vicinity of equilibrium points with good quality.

Note 2: In order to make the control input (Eq. (3.5)) smoother, especially near the equilibrium point, the terms that contain $p$ in their denominator are neglected. As will be shown in the simulation Section, this simplification leads to more implementable control inputs and still provides GUUB of the states. 
Depending on the magnitude of $p$ and the value chosen for $t h p_{0}$, two different cases occur during simulations:

— for $|p|>t h p_{0}$

$$
\begin{gathered}
u_{1}=\frac{-\alpha_{3} k_{r} q r}{k_{p}}-k_{p} p-\alpha_{1} q r-d_{p} \\
u_{2}=-k_{q} q+\frac{d}{c+d} \frac{k_{p} k_{r}}{\alpha_{3}} \frac{r}{p}-\alpha_{2} p r-d_{q} \\
- \text { for }|p| \leqslant t h p_{0} \\
u_{1}=\frac{-\alpha_{3} k_{r} q r}{k_{p}}-k_{p} p-\alpha_{1} q r-d_{p} \\
u_{2}=-k_{q} q-\alpha_{2} p r-d_{q}
\end{gathered}
$$

The above controllers are extended forms of LSB. Therefore, they will be called ELSB. In order to verify the analytical results, several simulations are carried out, and the results are presented in the next Section.

\section{Simulations}

The system and controller parameters are presented in Table 1. $c$ and $d$ are selected as 1 and -0.94 to -0.90 , respectively.

Table 1. System and controller parameters

\begin{tabular}{|c|c|c|c|}
\hline $\begin{array}{c}\text { Initial conditions } \\
{[\mathrm{deg} / \mathrm{s}]}\end{array}$ & $\begin{array}{c}\text { Boundary layer } \\
\text { thickness }[\mathrm{deg} / \mathrm{s}]\end{array}$ & $\begin{array}{c}\text { Controller } \\
\text { coefficients }\end{array}$ & $\begin{array}{c}\text { Moments of inertia } \\
{\left[\mathrm{kg} \mathrm{m}^{2}\right]}\end{array}$ \\
\hline \hline$p_{0}=8$ & & $k_{p}=0.05$ & $J_{x}=449.5$ \\
\hline$q_{0}=-6$ & $t h p_{0}=0.1$ & $k_{q}=0.1$ & $J_{y}=264.6$ \\
\hline$r_{0}=7$ & & $k_{r}=0.1$ & $J_{z}=312.5$ \\
\hline
\end{tabular}

Three scenarios are considered for simulation. These scenarios are presented in Table 2 .

Table 2. Simulation scenarios

\begin{tabular}{|c|c|c|}
\hline Scenario & Controller & Disturbance \\
\hline \hline First scenario & LSB & step \\
\hline Second scenario & ELSB & step \\
\hline Third scenario & LSB & sinusoidal \\
\hline
\end{tabular}

A comparison of the first and second scenarios analyzes the capabilities of the LSB and ELSB in dealing with a large step disturbance. A comparison of the first and third scenarios shows severity of the step disturbance compared to the sinusoidal disturbance.

\section{First scenario}

The component of disturbances exerted on the unactuated axis is considered as the following step function

$$
d_{r}=\frac{1}{J_{z}}
$$

Therefore, the external disturbance $\left(T_{d r}\right)$ exerted on the unactuated axis is $1 \mathrm{Nm}$. 
As stated in Assumption 1, the disturbances exerted on the actuated states are not important as long as they are known. Therefore, they are not considered here.

The LSB response is illustrated in Figs. 1 and 2.

As illustrated in Fig. 1, LSB is not able to provide GUUB for the states. The reason can be explained by Eq. (2.22). The controller tries to make the origin an asymptotically stable equilibrium for the closed-loop system. However, due to the presence of a large step disturbance on the unactuated axis, $r$ increases in an unacceptable way. This important example shows the adverse effects of not considering large non-matched disturbances in the controller design. Due to the non-smooth nature of the control inputs, a jump in the control moment is observed (Fig. 2).

Note 3: In order to reduce the adverse effects of sudden changes in the control moments and to make them more implementable, thickness of the boundary layer should increase, at the expense of less response quality.

\section{Second scenario}

In order to provide GUUB, the ELSB is used. The response and control moments of this controller are illustrated in Figs. 3 and 4:

According to Fig. 3, the ELSB makes the closed-loop system GUUB. As stated in Note 2, the simplifications have led to implementable control moments. At the same time, the states are bounded.

\section{Third scenario}

It is assumed that the following sinusoidal disturbance is exerted on the unactuated axis

$$
d_{r}=\frac{1}{J_{z}} \sin \left(\frac{2 \pi}{50} t\right)
$$

In comparison to Eq. (4.1), the amplitudes of the disturbances are the same.

The results of the simulation are shown in Figs. 5 and 6.

A comparison of Fig. 1 and Fig. 5 shows that the closed-loop system is bounded for the sinusoidal disturbance. However, for the step disturbance, the response of the unactuated axis becomes unbounded.

Finally, it can be concluded that the LSB makes the origin asymptotically stable for the disturbance-free closed-loop system. Therefore, in the absence of non-matched disturbances, the origin is asymptotically stable. However, this controller will not provide satisfactory performance when large non-matched disturbances are considered. On the other hand, the ELSB attenuates the effects of non-matched disturbances and makes the closed-loop system GUUB.

\section{Conclusion}

A mechanical system is underactuated when the number of independent control inputs is less than the number of degrees of freedom to be controlled. The presence of uncontrollable modes in their linearized models prevents them from being smooth state feedback stabilizable. The problems increase when disturbances, especially of the non-matched type, enter into the problem. In this paper, non-smooth time-invariant state feedback control laws have been obtained that made the origin asymptotically stable for underactuated spacecraft. Then, these control laws have been extended to make the closed-loop system GUUB. Simulation results have been presented to verify the analytical solutions. 

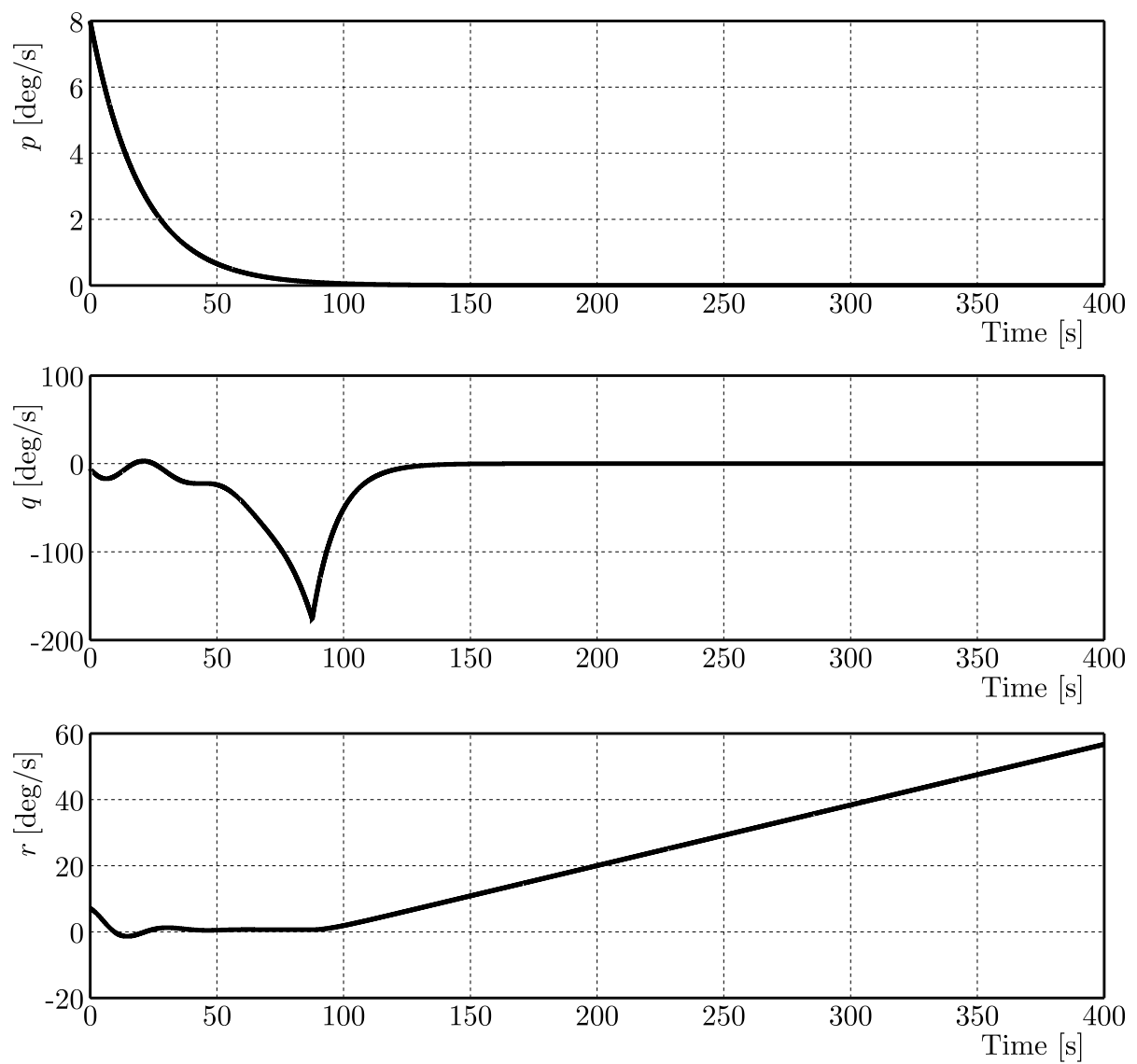

Fig. 1. Response of the LSB (first scenario)
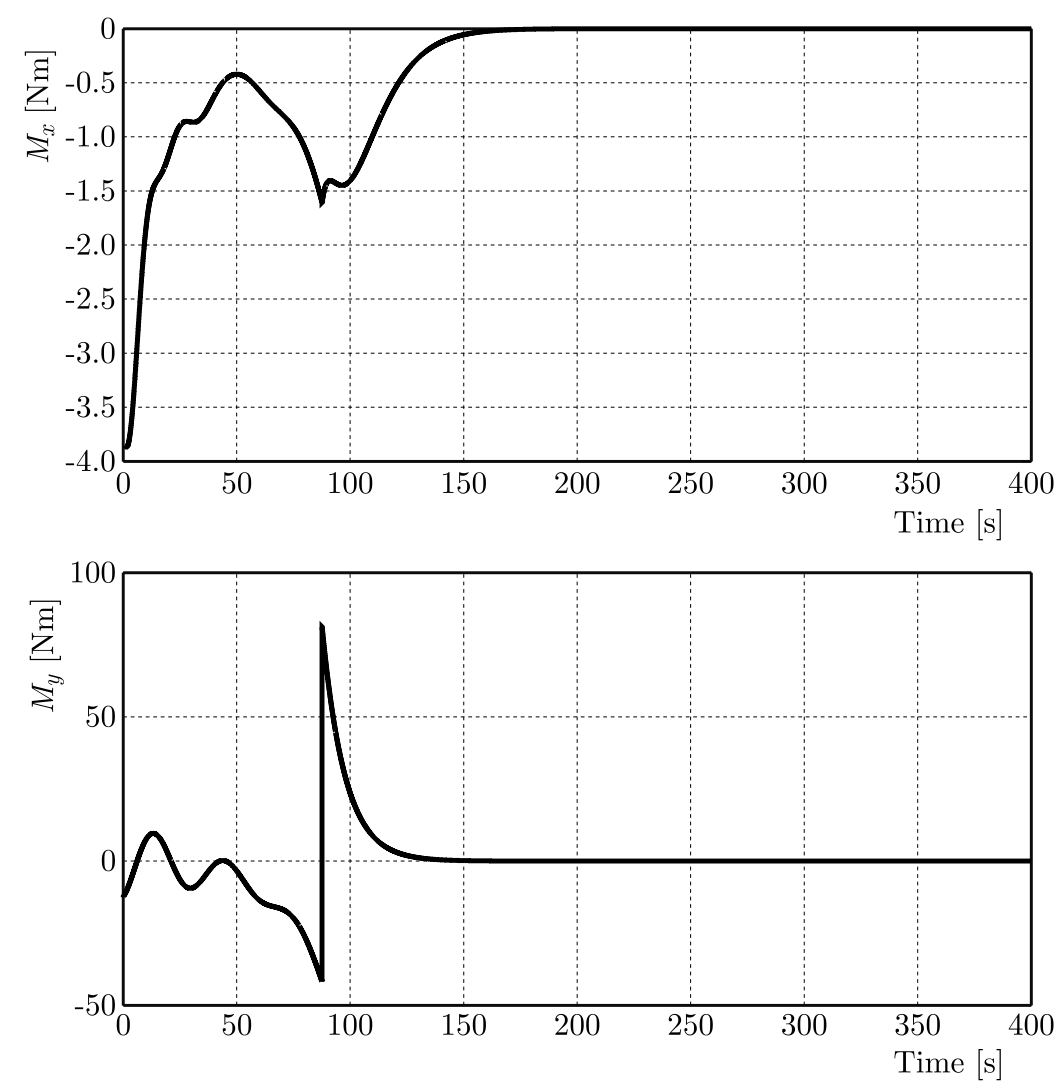

Fig. 2. Control moments 

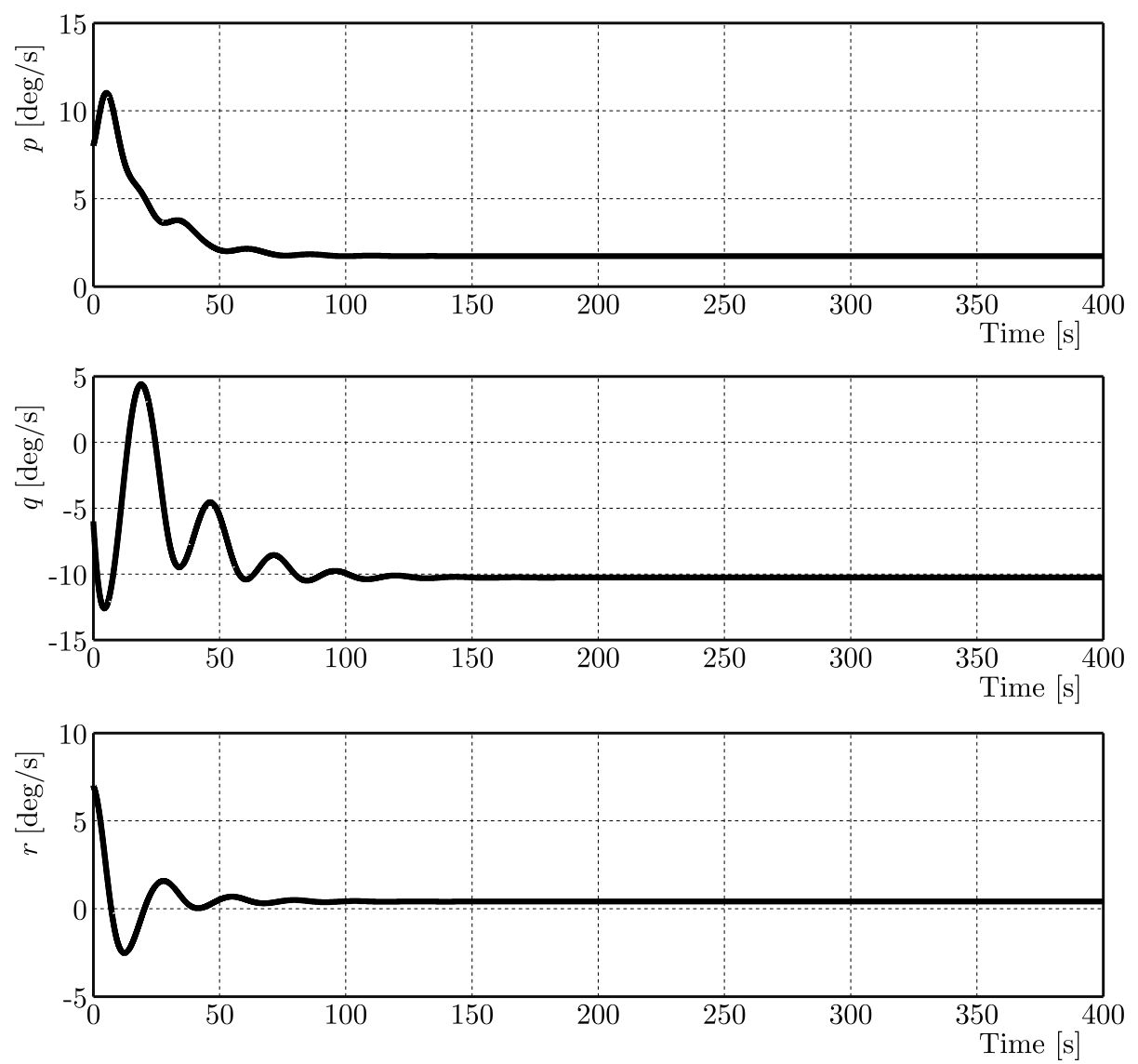

Fig. 3. Response of the ELSB (second scenario)
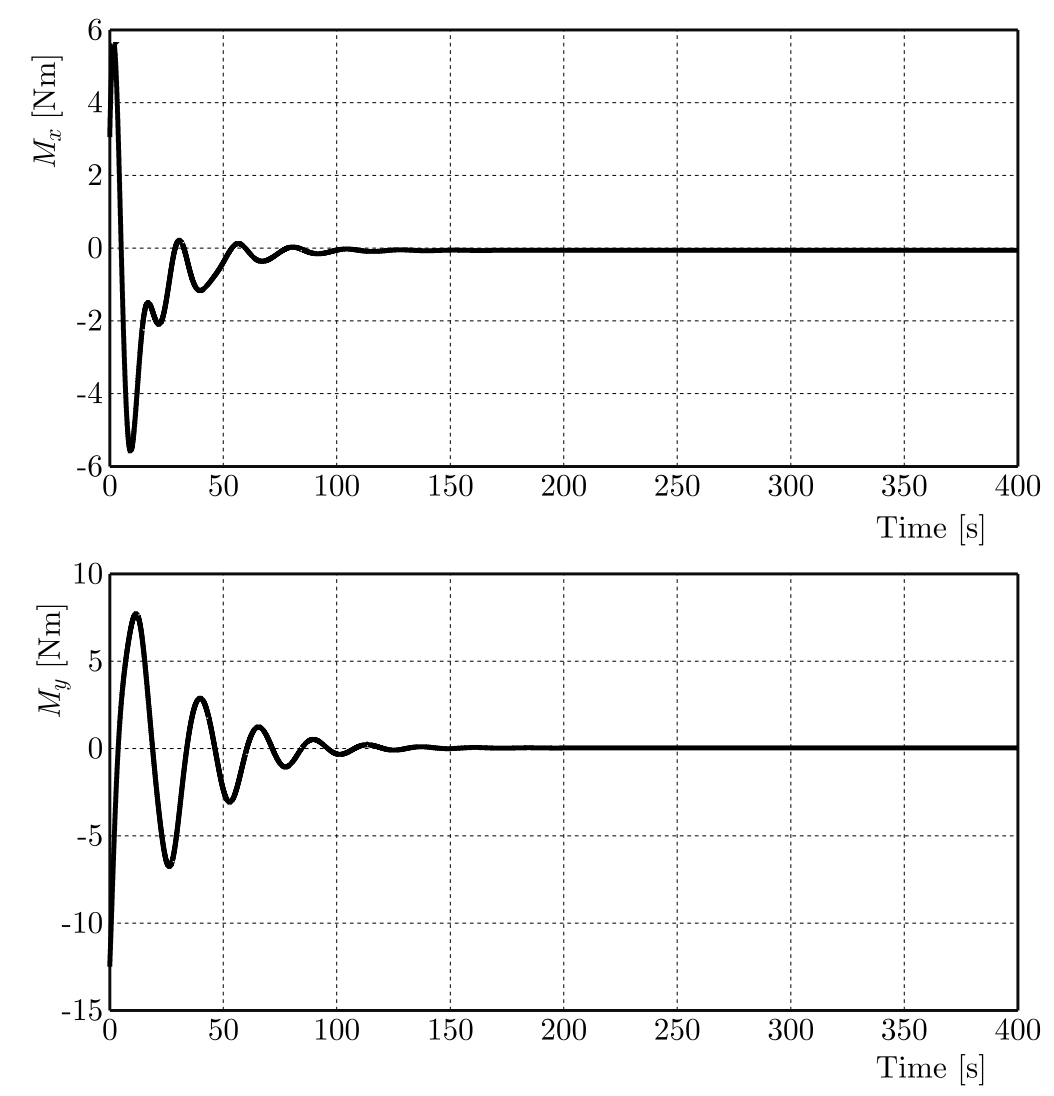

Fig. 4. Control moments 

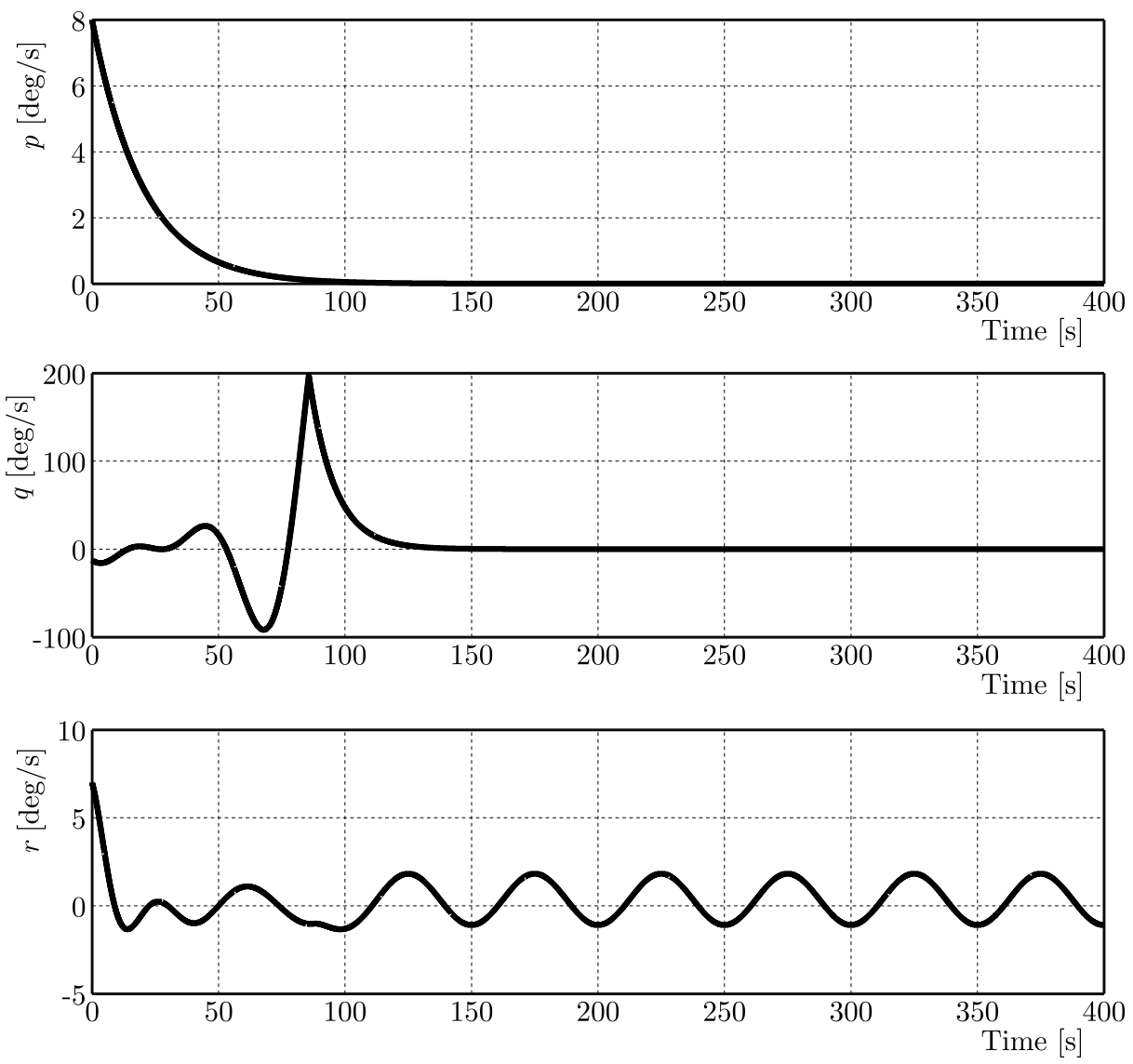

Fig. 5. Response of the LSB (third scenario)
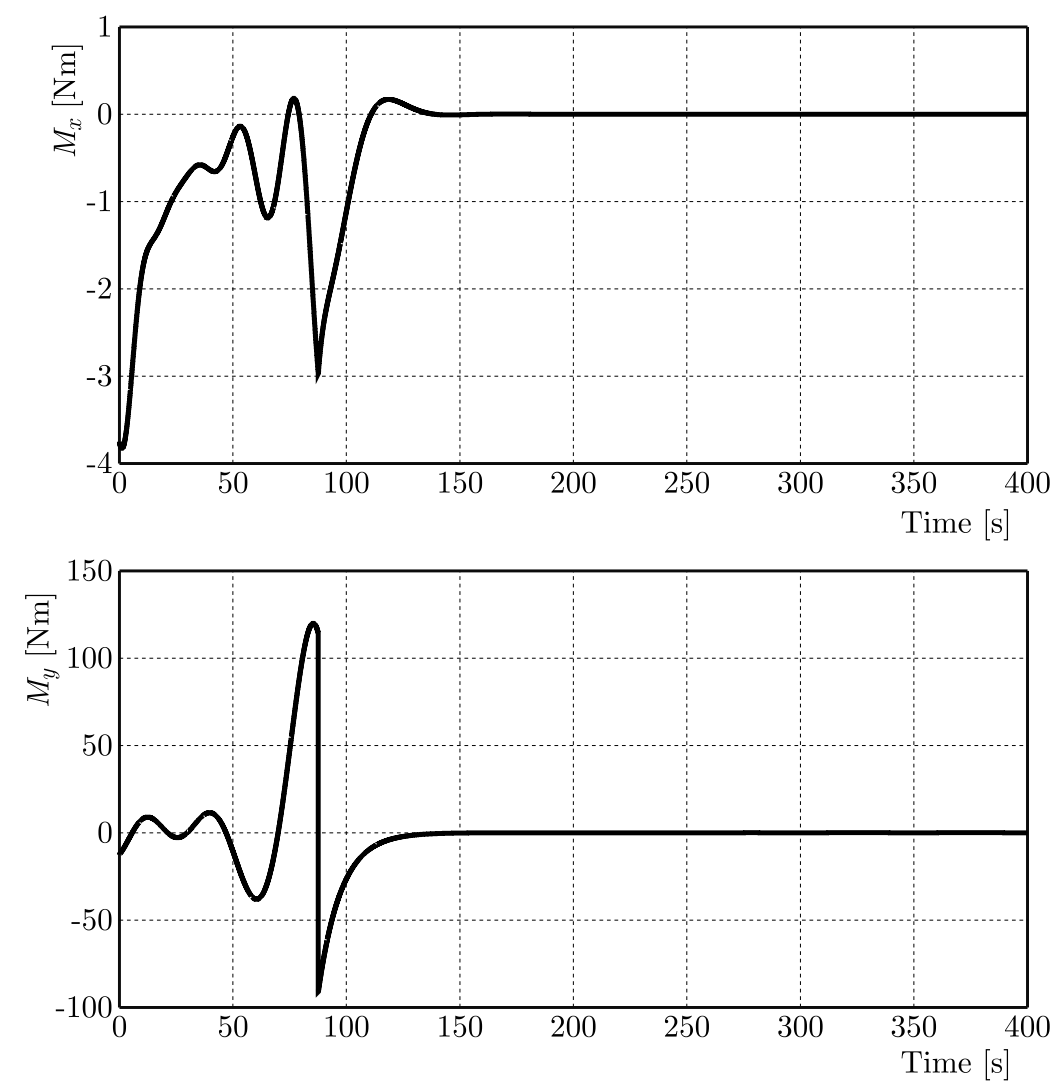

Fig. 6. Control moments 


\section{References}

1. Aneke N.P.I., 2003, Control of Underactuated Mechanical Systems, Eindhoven: Technische Universität Eindhoven, DOI: 10.6100/IR559509

2. Astolfi A., Rapaport A., 1998, Robust stabilization of the angular velocity of a rigid body, Systems and Control Letters, 34, 5, 257-264, DOI: 10.1016/S0167-6911(98)00031-0

3. Choukchou-Braham A., Cherki B., Djemai M., Busawon K., 2013, Analysis and Control of Underactuated Mechanical Systems, Springer, DOI: 10.1007/978-3-319-02636-7

4. Fantoni I., Lozano R., 2002, Nonlinear Control for Underactuated Mechanical Systems, Springer-Verlag London, Communication and Control Engineering, ISBN: 978-1-4471-1086-6, DOI: 10.1007/978-1-4471-0177-2

5. Floquet T., Perruquetti W., Barbot J.P., 2000, Angular velocity stabilization of a rigid body via VSS control, Journal of Dynamic Systems, Measurement, and Control, 122, 4, 669-673, DOI:10.1115/1.1316787

6. Karami M.A., Sassani F., 2007, Nonlinear attitude control of an underactuated spacecraft subject to disturbance torques, Proceedings of the American Control Conference, 3150-3155, DOI: 10.1109/ACC.2007.4283042

7. Khalil H., 2000, Nonlinear Systems, Prentice Hall, Third Edition, ISBN: 0-13-067389-7

8. LiU Y., Yu H., 2013, A survey of underactuated mechanical systems, IET Control Theory and Applications, 7, 7, 921-935, DOI: 10.1049/iet-cta.2012.0505

9. Olfati-Saber R., 2001, Nonlinear control of underactuated mechanical systems with application to robotics and aerospace vehicles, Ph.D. Thesis, MIT University

10. Reyhanoglu M., 1996, Discontinuous feedback stabilization of the angular velocity of a rigid body with two control torques, Proceedings of the 36th IEEE Conference on Decision and Control, 3, 2692-2694, DOI: 10.1109/CDC.1996.573511

11. Reyhanoglu M., Cho S., MCClamroch H.N., 2000, Discontinuous feedback control of a special class of underactuated mechanical systems, International Journal of Robust and Nonlinear Control, 10, 4, 265-281, DOI: 10.1002/(SICI)1099-1239(20000415)10:4<265::AID-RNC470>3.0.CO;2-N

12. Sidi M.J., 2000, Spacecraft Dynamics and Control: A Practical Engineering Approach, Cambridge Aerospace Series 7, ISBN: 0-521-55072-6

13. Spong M.W., 1998, Underactuated mechanical systems, Control Problems in Robotics and Automation, Lecture Notes in Control and Information Sciences, 230, 135-150, DOI: $10.1007 / \mathrm{BFb} 0015081$

14. WANG D., JiA Y., Jin L., Xu S., 2013, Control analysis of an underactuated spacecraft under disturbance, Acta Astronautica, 83, 44-53, DOI: 10.1016/j.actaastro.2012.10.029

15. Zhang H.H., Wang F., Trivailo P.M., 2008, Spin-axis stabilization of underactuated rigid spacecraft under sinusoidal disturbance, International Journal of Control, 81, 12, 1901-1909, DOI: 10.1080/00207170801930217 